\title{
Cortical activation during attention to sound in autism spectrum disorders.
}

\section{$\operatorname{AUTHOR}(S)$ :}

Funabiki, Yasuko; Murai, Toshiya; Toichi, Motomi

\section{CITATION:}

Funabiki, Yasuko ...[et al]. Cortical activation during attention to sound in autism spectrum disorders.. Research in developmental disabilities 2012, 33(2): 518-524

\section{ISSUE DATE:}

2012-03

URL:

http://hdl.handle.net/2433/152542

\section{RIGHT:}

(C) 2011 Elsevier Ltd.; This is not the published version. Please cite only the published version.; この論文は出版社版でありません。引用の際に は出版社版をご確認ご利用ください。 


\title{
Cortical activation during attention to sound in autism spectrum disorders
}

\author{
Yasuko Funabiki* $^{1,2}$, Toshiya Murai ${ }^{2}$, and Motomi Toichi ${ }^{1}$
}

${ }^{1}$ School of Human Health Science, Kyoto University, 53 Kawaharacho, Shogoin, Sakyo-ku, Kyoto 606-8507, Japan.

2 Department of Psychiatry, Graduate School of Medicine, Kyoto University, 54 Kawaharacho, Shogoin, Sakyo-ku, Kyoto 606-8507, Japan.

*Correspondence author: Yasuko Funabiki, Department of Psychiatry, Kyoto University Hospital, 54 Kawaharacho, Shogoin, Sakyo-ku, Kyoto 606-8507, Japan. E-mail: funaysk@kuhp.kyoto-u.ac.jp Tel: +81-75-751-3373; Fax: +81-75-751-4212. 


\begin{abstract}
Individuals with autism spectrum disorders (ASD) can demonstrate hypersensitivity to sounds as well as a lack of awareness of them. Several functional imaging studies have suggested an abnormal response in the auditory cortex of such subjects, but it is not known whether these subjects have dysfunction in the auditory cortex or are simply not listening. We measured changes in blood oxygenated hemoglobin $(\mathrm{OxyHb})$ in the prefrontal and temporal cortices using near-infrared spectroscopy during various listening and ignoring tasks in 11 ASD and 12 control subjects. Here we show that the auditory cortex in ASD subjects responds to sounds fully during attention. OxyHb in the auditory cortex increased with intentional listening but not with ignoring of the same auditory stimulus in a similar fashion in both groups. Cortical responses differed not in the auditory but in the prefrontal region between the ASD and control groups. Thus, unawareness to sounds in ASD could be interpreted as due to inattention rather than dysfunction of the auditory cortex. Difficulties in attention control may account for the contrary behaviors of hypersensitivity and unawareness to sound in ASD.
\end{abstract}

Keywords: Autism, pervasive developmental disorder, auditory, attention, near-infrared spectroscopy, Asperger. 


\section{Introduction}

Autism spectrum disorder (ASD) is characterized by qualitative abnormalities of reciprocal social interaction, together with a restricted, stereotyped, repetitive repertoire of interests and activities. Infants with ASD may be first noticed to be unaware of being called, sometimes misunderstood as hearing disorders. In children with ASD, atypical auditory processing, such as auditory filtering difficulties or sensory under-responsiveness, is associated with academic underachievement (Ashburner, Ziviani, \& Rodger, 2008). As they grow up, the unawareness decreases, but it usually persists more or less throughout life. On the other hand, autistic persons have been reported to show hypersensitivity to noise (Harrison \& Hare, 2004; Leekam, Nieto, Libby, Wing, \& Gould, 2007). Thus, behavioral response to sounds in ASD is inconsistent.

Several researchers have investigated this phenomenon. Behavioral and audiological studies have shown that behavioral responses to sounds are not associated with hypersensitivity of the auditory pathways but with difficulties in the higher cortical processing systems (Gomes, Rotta, Pedroso, Sleifer, \& Danesi, 2004; Tharpe et al, 2006). Electrophysiological findings have indicated that sensory sound processing was intact but that involuntary orienting was affected (Ceponiene et al., 2003), and that the early left frontal component was abnormal, not the temporal component (Gomot, Giard, Adrien, Barthelemy, \& Bruneau, 2002). Taken together, these findings suggest that the unawareness to sounds in ASD is not from dysfunction of the auditory pathway itself.

Cortical response to sounds in the auditory area in autism has also been measured. Studies using positron emission tomography have shown cortical hypo-activation with passive listening to speech-like sounds in autism with mental retardation both in adults 
(Boddaert et al., 2003) and children (Boddaert et al., 2004). Gervais et al (2004)

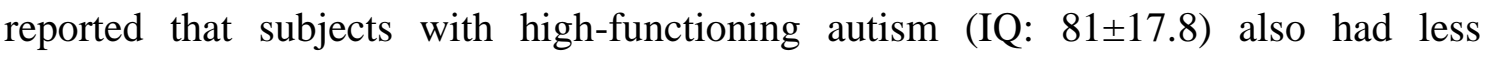
activation in the superior temporal sulcus region with functional magnetic resonance imaging (fMRI) during passive listening to vocal sounds, and that recall of the presented voices was also impaired in subjects who had hypo-activation of that region.

Meanwhile, it has been established that attention modulates the activities of the auditory cortex (Pugh et al., 1996; Grady et al., 1997; Jäncke, Mirzazade, \& Shah, 1999; Petkov et al., 2004; Rinne et al., 2007; Woods et al., 2009). When greater attention to sounds is demanded, there is more activation of the temporal lobe. Therefore, the findings of decreased response in the temporal lobe in autistic subjects may simply reflect decreased attention to sounds in autism, rather than impairment of auditory areas per se.

The purpose of this study is to clarify whether the hypo-activation in ASD is due to dysfunction of the auditory cortex or merely due to inattention to voices. Thus, we investigated whether the auditory cortex is activated while ASD participants listened to sounds intentionally. The use of the passive listening paradigm that was applied in the previous studies would not be sufficient to elucidate this issue. We recorded cortical response to sound during modulation of attention using near-infrared spectroscopy (NIRS) in ASD and control subjects.

\section{Materials and methods}

\subsection{Participants}

$<<$ Table 1 around here $>>$ 
The characteristics of participants are shown in Table 1. Informed consent was obtained from all the participants. NIRS can measure real-time brain response without averaging repeated trials, which enables monitoring of brain activity during on-the-spot attention. To control the level of attention to sound stimuli, it was essential that the subjects were unimpaired in basic verbal ability. Thus, we selected participants without language delay or mental retardation but with distinct ASD symptoms, limiting the diagnoses of subjects to Asperger's disorder or pervasive developmental disorder (PDD) not otherwise specified by the definition of the Diagnostic and Statistical Manual of Mental Disorders - Fourth Edition - Text Revision (DSM-IV-TR) (American Psychiatric Association, 2000). Both of these belong to the category of ASD or PDD. Autistic disorder by definition was excluded because of the language delay.

\subsection{Sound files preparation}

Since previous researchers have reported poor responses to voices compared with non-verbal sounds in autistic subjects (Ceponiene et al., 2003; Gervais et al., 2004), we focused on the cortical response to voices, especially those of meaningful stories. We also prepared tone sounds just for comparison, but since tones and meaningful stories are very different, we inserted step-by-step transition stimuli: vowels (each tone sound was replaced with a vowel) and a meaningless syllable sequence (reverse syllable reading of the meaningful story). Sound files were set to 30 seconds. The first file was a succession of two kinds of pure tones. The duration of each tone was $100 \mathrm{~ms}$ and the file included 45 tones. Most sounds were $1-\mathrm{kHz}(83 \%)$, with $2-\mathrm{kHz}$ tones $(17 \%)$ randomly presented to avoid boredom. The second file was a succession of two vowels "e" and "i" by female voice. The presentation was the same as the first file; the sound 
"e" took the place of the $1-\mathrm{kHz}$ tone. These files were made with MATLAB 6.0. The third file was a meaningless syllable sequence, made by reading syllables of the fourth files in reverse order. The fourth files were simple stories with 30 words read by a female voice.

\subsection{Settings of NIRS}

We set up 32 probes. Each probe was located at $3 \mathrm{~cm}$ intervals. Spatial resolution is $20-30 \mathrm{~mm}$. For monitoring the activation of the prefrontal cortex, we used the channels located at $15 \mathrm{~mm}$ above Fp1 and Fp2 of the international 10-20 system for electrode placement. For that of the auditory cortex, we chose the central channel among the activated ones lower or posterior to F7 on the left side and F8 on the right side. We used the same channels through all the recordings in each subject. The recorded waves were stored on a computer with $130 \mathrm{~ms}$ intervals. Details on data acquisition are written in the paper by Matsuda and Hiraki (2006).

\subsection{Data recording}

Subjects sat in front of a display with a simple meaningless screensaver, and put on a helmet-like apparatus with a multiple-channel NIRS (up to 46 channels, OMM-3000; Shimadzu Co., Japan). Two speakers were placed on both sides of the subject. The average intensity of the presented sounds was set at $75 \mathrm{~dB}$ sound pressure level. After establishing a stable baseline on NIRS, we started the recording in each session. We conducted six sessions using these four types of sound files. In the first two sessions, we played the tone file. In one session, subjects were asked to listen carefully. In the other session, they were asked not to listen but just to ignore the played file. The third session 
was intentional listening to a sequence of vowels. The fourth session was also intentional listening to the reverse reading of a meaningful story. In each of the remaining two sessions, we presented a different file of meaningful short stories. Similar to the first two sessions, one was the listening task and the other was the ignoring task. Thus, we measured changes in blood oxygenated hemoglobin in the prefrontal and temporal cortices during various listening and ignoring tasks.

We monitored waves of oxygenated (oxy) and deoxygenated (deoxy) hemoglobin $(\mathrm{Hb})$ by NIRS in the prefrontal and auditory cortices. Examples of the waves are shown in Figure 1. In general, oxyHb in the temporal cortex increased when subjects listened to sounds or voices, but oxyHb in the prefrontal cortex decreased during many tasks.

$<<$ Figure 1 around here $>$

\subsection{Data analyses}

We computed the mean value of oxyHb between 10 to 30 seconds from the start of each stimulus, and then subtracted the averaged baseline (calculated from data of 10 seconds before stimuli) to obtain oxyHb changes with the stimuli. Then we calculated averages and standard errors by tasks, attention states, sides and groups (see Figure 2). We compared the changes of oxyHb using ANOVA by SPSS 19.0.

$<<$ Figure 2 around here $>>$

\section{Results}

\subsection{Cortical response to sounds}

First, we compared cortical responses between "listening" and "ignoring" to the same kinds of auditory stimuli. Since the ignoring state was only present in the two 
tasks of tone and story, we performed a $2 \times 2 \times 2$ mixed randomized-repeated ANOVA on changes of oxyHb for each of the two stimuli sets and each of the temporal and prefrontal cortices. The factors were side, attention state and group. Eleven ASD patients were compared with 12 controls. OxyHb was measured on the left and right sides of each person and when listening to and ignoring sound stimuli. In the temporal cortex, we found only main effects of attention in both stimuli of tone and story ( $p=$ 0.012 in the stimuli of tone, $p<0.001$ in the stimuli of story) and no interactions. The highly reliable main effects show that individuals with ASD as well as controls have an apparent $\mathrm{xxyHb}$ increase when they are requested to listen intentionally. In the prefrontal cortex, there was a significant three-way interaction among side, attention state and group $(p=0.0026)$ in the story task. We also found a two-way interaction between side and attention state $(p=0.032)$ in this task. Therefore, the interactions were compared separately for the two groups. Only for the ASD group was the interaction statistically reliable $(p=0.001)$, whereas there was no interaction for controls $(p=$ 0.426). These results indicate that individuals with ASD might have an abnormal pattern of hemispheric laterality switching when they attempt to regulate their attention to auditory stimuli. However, as the number of participants was small, further investigation is needed on this issue. Neither main effects nor interactions were detected in the tone task in the prefrontal cortex.

We then compared the differences of responses between three pairs of tasks (tone vs. voice, voice vs. reverse, and reverse vs. story) with the intentional listening state. We carried out $2 \times 2 \times 2$ mixed randomized-repeated ANOVAs with the factors of side, task and group. Neither main effects of group nor interactions involving the factor of group were revealed to be significant, indicating that cortical responses to various types of 
sounds do not differ between ASD and controls as long as they listened intentionally. Although they were not our main focus, the statistical results, which do not involve the factor of group, in these analyses were as follows: We found a significant main effect of stimulus when we compared the stimuli of reverse and story $(p=0.0013)$ without any interaction in the temporal cortex. This main effect indicates that an oxyHb increase in the auditory area is induced when subjects listen to meaningful stories but not to meaningless syllable sequences. There were no main effects or interactions in other comparisons in the temporal cortex. In the prefrontal cortex, in contrast, there were main effects of side, showing a larger oxyHb decrease on the left side, in the comparisons between the two pairs of tone vs. voice $(p=0.038)$ and voice vs. reverse ( $p$ $=0.035)$. Also we found an interaction of task and side $(p=0.040)$ when we compared the tasks of reverse and story.

\subsection{Recall test}

$<$ Figure 3 around here $>$

After each of the story sessions, subjects were asked to recall the story. We counted the recalled words. Interestingly, individuals with ASD remembered some parts of the story even when they tried to ignore it (Figure 3). The correctly recalled word numbers were analyzed using a $2 \times 2$ mixed randomized-repeated ANOVA with the factors of group and attention. We found an interaction between the two factors $(p=0.012)$ and also main effects of attention $(p<0.001)$ and of group $(p=0.045)$. Therefore, we compared group differences separately for the two attention states. When subjects listened intentionally, the number of recalled words did not differ between the two groups $(p=0.726)$. However, when subjects were instructed to ignore the story, ASD 
subjects recalled more words than controls $(p=0.003)$. This result is interesting because the temporal cortex was not activated in ASD subjects in the story-ignoring condition, which is essentially the same as control subjects (see Figure 2).

\section{Discussion}

The phenomenon that attention modulates activation in the auditory cortex has been established in healthy subjects (Pugh et al., 1996; Grady et al., 1997; Jäncke et al., 1999; Petkov et al., 2004; Rinne et al., 2007; Woods et al., 2009). We found that it is also the case in autistic subjects. By contrast, previous studies applying a passive listening paradigm in autistic subjects demonstrated hypo-activation of the temporal cortex (Boddaert et al., 2003; Boddaert et al., 2004; Gervais et al., 2004). The intentional listening condition in our current study appeared to cause the same level of activation of the auditory cortex in ASD as in controls. Thus, the unawareness of autistic participants could be interpreted as due to inattention rather than cortical dysfunction.

Deactivations in the prefrontal cortex are, to a certain extent, general phenomena during various types of cognitive task application. In the studies using NIRS, these phenomena have been reported in the tasks of reading aloud (Fallgatter, Muller, \& Strik, 1998), reaching (Shimada, Hiraki, Matsuda, \& Oda, 2004), and video games (Matsuda et al, 2006). F-MRI studies have also detected these task-induced deactivations, which are thought to reflect an interruption of mental activities during rest, proposed as the “default mode network" (DMN) (Raichle et al., 2001; Gusnard and Raichle, 2001; Mayer, Roebroeck, Maurer, \& Linden, 2010). In autism, deactivation in the default mode network was suggested to be different from controls, although activities in the task-positive network (Fox et al., 2005) and the task performance did not differ 
(Cherkassky, Kana, Keller, \& Just, 2006; Kennedy, Redcay, \& Courchesne, 2006; Kennedy, \& Courchesne, 2008). These findings are compatible with our results, in which the prefrontal cortex showed task-induced deactivation with a different pattern from controls, that is, hemispheric laterality switching of the deactivation during regulating attention to sounds only in ASD, while the auditory area (which is considered to be a major constituent of the task-positive network) and task performance of the word recall during attention did not differ. Furthermore, a recent study showed that motivation normalizes the atypical pattern of the task-related DMN deactivation in attention deficit/hyperactivity disorder (ADHD) (Liddle et al, 2011). Thus, attention might compensate for the attenuated cortical response of the DMN as well as of the auditory cortex in ASD and/or ADHD in our study.

Although we cannot clarify the reason for the increased recalls during the ignoring condition in ASD, difficulties in attention control may account for it. In the listening condition, the equal-level increase of oxyHb in the auditory cortex and also the same level of word recalls in both groups indicate that they followed the instruction. In the ignoring condition, the lack of oxyHb increase in both groups also suggests that they followed the direction. However, ignoring the audible story intentionally is more difficult than intensive listening for autistic subjects. That is, even if they tried to ignore the story, which might lead to the lack of oxyHb increase, they might not be able to eliminate the audible story intentionally, resulting in partial memory even without conscious intention. This phenomenon may possibly relate to the hypersensitivity to sounds observed in ASD. The difficulty in regulating attention to sounds in ASD has already been pointed out by an event-related potential study (Ceponiene et al., 2003). Also, a recent behavioral observational study has shown that individuals with ASD 
often have difficulty paying attention (Funabiki, Kawagishi, Uwatoko, Yoshimura, \& Murai, 2011). These reports are in concordance with our results. Furthermore, an electrophysiological study showing that children with autism had left frontal rather than temporal cortical dysfunction is also supportive of our results of cortical response patterns (Gomot et al, 2002).

\section{Conclusion}

The current study indicates that the auditory cortex in ASD subjects responds to sounds and voices as long as the subjects attend to them. The core problem of their apparent unawareness of voices would be explained by hypothesizing the existence of a deficit in regulating attention to voices. In the framework of this hypothesis, the contradictory observation that ASD subjects also show hypersensitivity to sounds is also explainable. We expect that the results of our study will contribute to our understanding of the struggles that ASD patients encounter in their lives, and eventually lead to the reduction of these difficulties through this better understanding by those around them.

\section{Acknowledgments}

This work was supported by a Grant-in-Aid for Young Scientists (B) (17730516). We would like to acknowledge all the participants, and thank the Osaka YMCA, Naomi Matsuura and Yukari Ise for help in recruiting the participants, and Kazuo Funabiki for help with preparing auditory stimuli. Finally, we sincerely thank Shimadzu Corporation for providing and setting up the NIRS.

All authors report no biomedical financial interests or potential conflicts of interest. 


\section{References}

American Psychiatric Association. (2000). Diagnostic and Statistical manual of Mental Disorders - Fourth Edition - Text Revision (DSM-IV-TR). Washington, DC: American Psychiatric Press.

Ashburner, J., Ziviani, J., \& Rodger, S. (2008). Sensory processing and classroom emotional, behavioral, and educational outcomes in children with autism spectrum disorder. American Journal of Occupational Therapy, 62, 564-73.

Boddaert, N. Belin, P., Chabane, N., Poline, J.B., Barthélémy, C., Mouren-Simeoni, M.C., et al. (2003). Perception of complex sounds: abnormal pattern of cortical activation in autism. American Journal of Psychiatry, 160, 2057-2060.

Boddaert, N., Chabane, N., Belin, P., Bourgeois, M., Royer, V., Barthelemy, C., et al. (2004). Perception of complex sounds in autism: abnormal auditory cortical processing in children. American Journal of Psychiatry, 161, 2117-2120.

Ceponiene, R., Lepistö, T., Shestakova, A., Vanhala, R., Alku, P., Näätänen, R., et al. (2003). Speech-sound-selective auditory impairment in children with autism: they can perceive but do not attend. The Proceedings of the National Academy of Sciences of the United States of America, 100, 5567-5572.

Cherkassky, V.L., Kana, R.K., Keller, T.A., \& Just, M.A. (2006). Functional connectivity in baseline resting-state network in autism. Neuroreport, 17, 1687-1690.

Fallgatter, A.J., Muller, Th.J., \& Strik, W.K. (1998). Prefrontal hypooxygenation during language processing assessed with near-infrared spectroscopy. Neuropsychobiology, $37,215-218$.

Fox, M.D., Snyder, A.Z., Vincent, J.L., Corbetta, M., Van Essen, D.C., \& Raichle, M.E. (2005). The human brain is intrinsically organized into dynamic, anticorrelated 
functional networks. Proceedings of the National Academy of Sciences of the United States of America, 102, 9673-9678.

Funabiki, Y., Kawagishi, H., Uwatoko, T., Yoshimura, S., \& Murai, T. (2011). Development of a multi-dimensional scale for PDD and ADHD. Research in Developmental Disabilities, 32, 995-1003.

Gervais, H., Belin, P., Boddaert, N., Leboyer, M., Coez, A., Sfaello, I., et al. (2004). Abnormal cortical voice processing in autism. Nature Neuroscience, 7, 801-802.

Gomes, E., Rotta, N.T., Pedroso, F.S., Sleifer, P., \& Danesi, M.C. (2004). Auditory hypersensitivity in children and teenagers with autistic spectrum disorder. Arquivos de Neuro-psiquiatria, 62, 797-801.

Gomot, M., Giard, M.H., Adrien, J.L., Barthelemy, C., \& Bruneau, N. (2002). Hypersensitivity to acoustic change in children with autism: electrophysiological evidence of left frontal cortex dysfunctioning. Psychophysiology, 39, 577-84.

Grady, C.L., Van Meter, J.W., Maisog, J.M., Pietrini, P., Krasuski, J., \& Rauschecker, J.P. (1997). Attention-related modulation of activity in primary and secondary auditory cortex. Neuroreport, 8, 2511-2516.

Gusnard, D.A., \& Raichle, M.E. (2001). Searching for a baseline; functional imaging and the resting human brain. Nature Reviews Neuroscience, 2, 685-694.

Harrison, J., \& Hare, D.J. (2004). Assessment of sensory abnormalities in people with autistic spectrum disorders. Journal of Autism and Developmental Disorders, 34, 727-730.

Jäncke, L., Mirzazade, S., \& Shah, N.J. (1999). Attention modulates activity in the primary and the secondary auditory cortex: a functional magnetic resonance imaging study in human subjects. Neuroscience Letters, 266, 125-128. 
Kennedy, D.P., Redcay, E., \& Courchesne, E. (2006). Failing to deactivate: Resting functional abnormalities in autism. Proceedings of the National Academy of Sciences of the United States of America, 103, 8275-8280.

Kennedy, D. P., \& Courchesne, E. (2008). The intrinsic functional organization of the brain is altered in autism. Neuroimage, 39, 1877-1885.

Leekam, S.R., Nieto, C.S., Libby, J., Wing, L. \& Gould, J. (2007). Describing the sensory abnormalities of children and adults with autism. Journal of Autism and Developmental Disorders, 37, 894-910.

Liddle, E.B., Hollis, C., Batty, M.J., Groom, M.J., Totman, J.J., Liotti, M., et al. (2011). Task-related default mode network modulation and inhibitory control in ADHD: effects of motivation and methylphenidate. Journal of Child Psychology and Psychiatry, 52, 761-71.

Matsuda, G., \& Hiraki, K. (2006). Sustained decrease in oxygenated hemoglobin during video games in the dorsal prefrontal cortex: A NIRS study of children. Neuroimage, 29, 706-711.

Mayer, J.S., Roebroeck, A., Maurer, K., \& Linden, D.E.J. (2010). Specialization in the default mode: Task-induced brain deactivations dissociate between visual working memory and attention. Human Brain Mapping, 31, 126-139.

Petkov, C. I., Kang, X., Alho, K., Bertrand, O., Yund, E.W., \& Woods, D.L. (2004). Attention modulation of human auditory cortex. Nature Neuroscience, 7, 658-663.

Pugh, K.R., Offywitz, B.A., Shaywitz, S.E., Fulbright, R.K., Byrd, D., Skudlarski, P., et al. (1996). Auditory Selective Attention: An fMRI investigation. Neuroimage, 4, $159-173$. 
Raichle, M.E., MacLeod, A.M., Snyder, A.Z., Powers, W.J., Gusnard, D.A., \& Shulman, G.L. (2001). A default mode of brain function. Proceedings of the National Academy of Sciences of the United States of America, 98, 676-682.

Rinne, T., Stecker, G.C., Kang, X., Yund, E.W., Herron, T.J., \& Woods, D.L. (2007). Attention modulates sound processing in human auditory cortex but not the inferior colliculus. Neuroreport, 18, 1311-1314.

Shimada, S., Hiraki, K., Matsuda, G., \& Oda, I. (2004). Decrease in prefrontal hemoglobin oxygenation during reaching tasks with delayed visual feedback: a near-infrared spectroscopy study. Cognitive Brain Research, 20, 480-490.

Tharpe, A.M., Bess, F.H., Sladen, D.P., Schissel, H., Couch, S., \& Scherv, T. (2006). Auditory characteristics of children with autism. Ear and Hearing, 27, 430-41.

Woods, D. L., Stecker, G.C., Rinne, T., Herron, T.J., Cate, A.D., Yund, E.W., et.al. (2009). Functional maps of human auditory cortex: effects of acoustic features and attention. PLoS One, 4, e5183. 


\section{FIGURE LEGENDS}

Figure 1. Examples of the recordings by near-infrared spectroscopy

A. Schematic drawing of recording locations. Gray circles indicate the location of channels. Fp1 in the international 10-20 system was the landmark of the probe setting, shown as a black circle in the figure. This example only shows the responses on the left side of a control subject, but the recordings on the right side were similar. B. Example of the responses to tones in the ignoring state. Black and gray lines indicate oxyHb and deoxyHb, respectively. Dashed and gray vertical lines indicate the start and the end of the stimuli, respectively. The duration was 30 seconds. C. Responses to the same tones in the listening state by the same subject. Temporal areas were activated by tones in the intentional listening state, but not in the ignoring state.

Figure 2. Analyses of temporal and prefrontal cortical responses to several sounds A. Locations of the channels used for the analyses. The channels $15 \mathrm{~mm}$ above the Fp1 on the left and Fp2 on the right side were selected for the analysis of the prefrontal cortex. The center of the activated channels in the auditory area was selected for the analysis of the temporal cortex. B. Examples of the responses to stories. Lines are shown as in Figure 1. C, D. Responses of the temporal and prefrontal cortices. Bars indicate the averages and error bars indicate the standard errors. Temporal areas in both control and ASD subjects had an oxyHb increase during listening to tones and stories, although the same sounds did not activate those areas in the ignoring state. The prefrontal cortex had an oxyHb decrease in many 
tasks in both groups. The difference between groups seen was only in the story task. The ASD group showed a laterality switch with attention.

Figure 3. Word numbers recalled after playback of stories.

Each story consisted of 30 words. Bars indicate the averages, and error bars indicate the standard errors. When subjects listened to the story, both groups recalled almost the same number of words. In the ignoring state, controls did not remember the story, but the ASD subjects recalled some parts of the story. 
$\Delta$

Controbled copy in

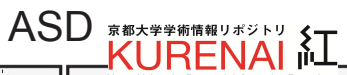

C Temporal

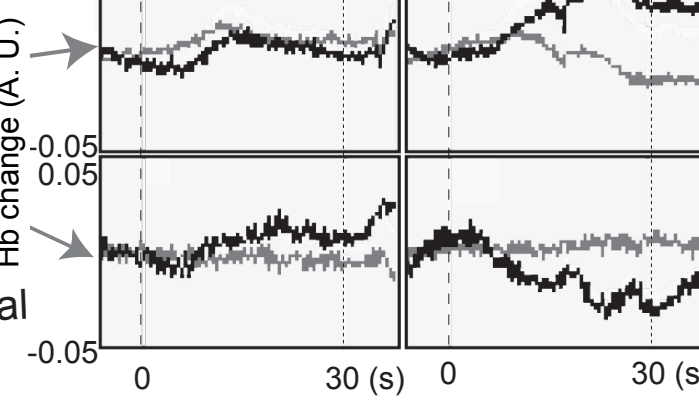

3
3
8
0
0
0
0
0
0

0.04

$-0.01$

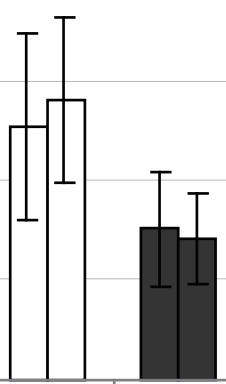

Side Group

L R L L R

R R L R
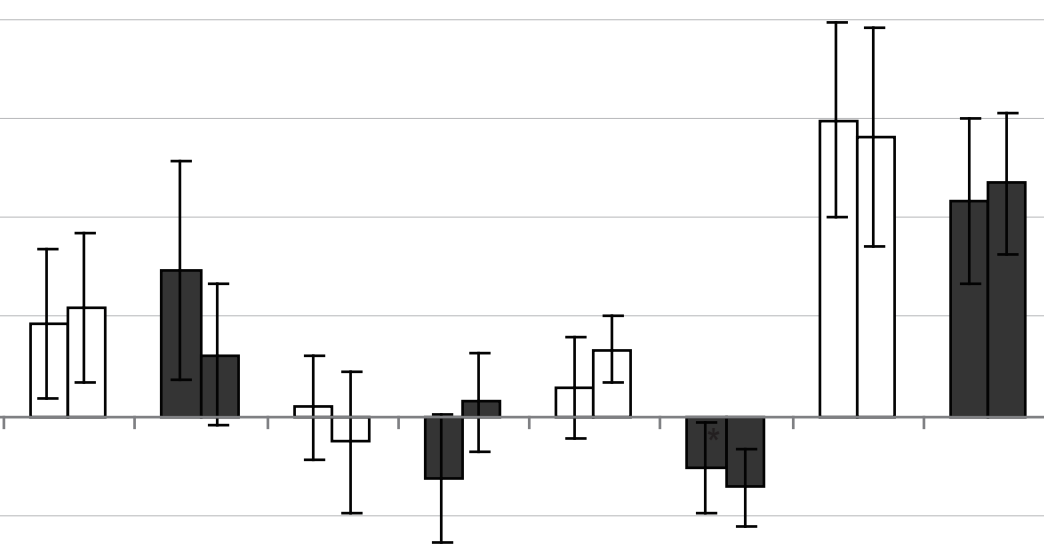
Attention Ignore

Task Tone Listen

D Prefrontal

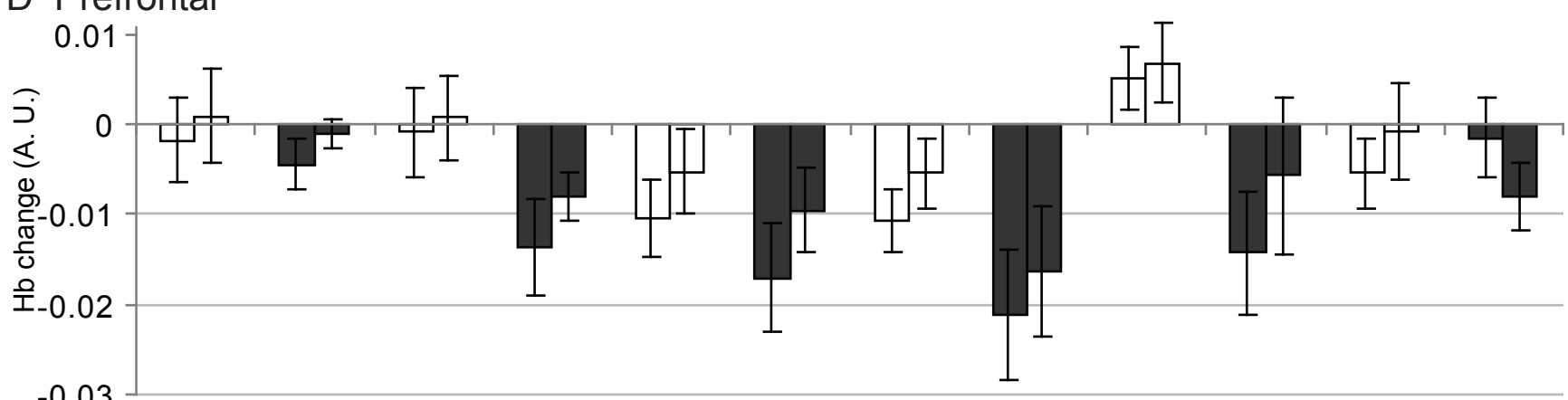
$-0.03$

Listen

Voice
Listen

Reverse

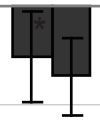

Ignore Listen

Story 


\section{A Self-archived copy in}

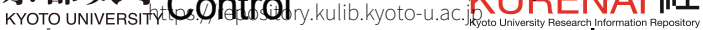

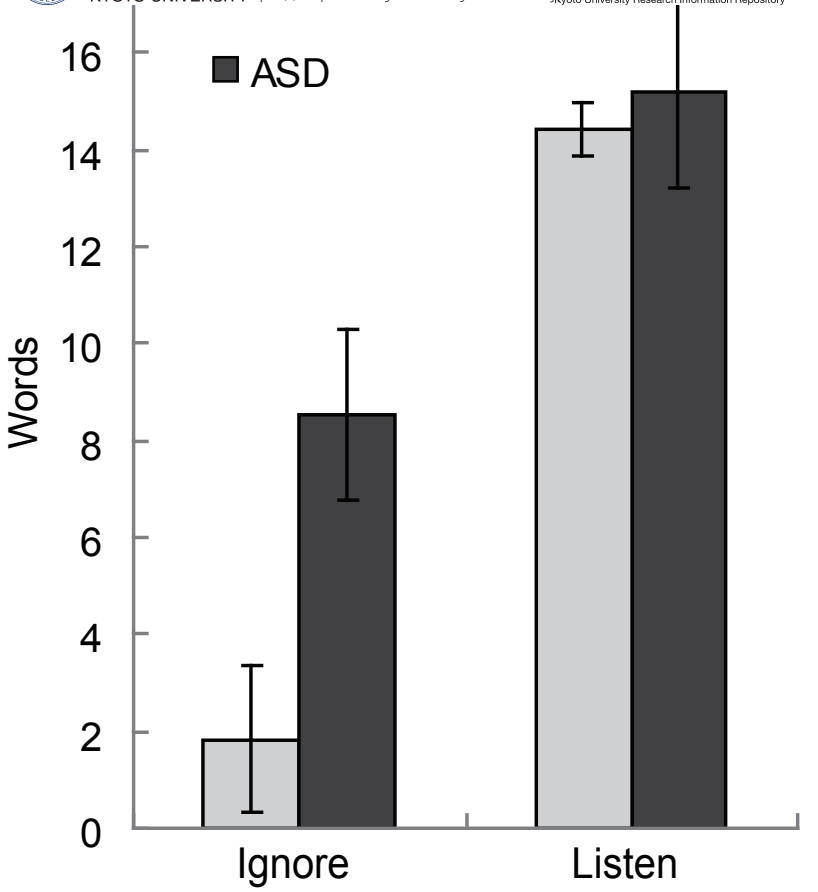


Table

\begin{tabular}{lccc} 
& ASD & Control & $P$ \\
\hline Number & 11 & 12 & \\
Male : Female & $10: 1$ & $10: 2$ & \\
Age & $16.8 \pm 6.1$ & $14.2 \pm 3.8$ & 0.22 \\
FIQ & $110.1 \pm 10.9$ & $102.9 \pm 13.6$ & 0.18 \\
VIQ & $112.5 \pm 12.5$ & $101.5 \pm 12.6$ & 0.048 \\
PIQ & $105.3 \pm 12.8$ & $103.8 \pm 15.0$ & 0.797 \\
\hline
\end{tabular}

Table 1. Characteristics of participants. Age and IQs are shown in means \pm standard variations. Group differences were analyzed using student $t$-tests. 